American Journal of Economics and Business Administration 2 (1): 27-34, 2010

ISSN 1945-5488

(C) 2010 Science Publications

\title{
Neuro-Based Artificial Intelligence Model for Loan Decisions
}

\author{
Shorouq Fathi Eletter, Saad Ghaleb Yaseen and Ghaleb Awad Elrefae \\ Department of Management Information Systems, \\ Faculty of Economics and Administrative Sciences, \\ Al-Zaytoonah University of Jordan, P.O. Box (130), Amman (11733), Jordan
}

\begin{abstract}
Problem statement: Despite the increase in consumer loans defaults and competition in the banking market, most of the Jordanian commercial banks are reluctant to use artificial intelligence software systems for supporting loan decisions. Approach: This study developed a proposed model that identifies artificial neural network as an enabling tool for evaluating credit applications to support loan decisions in the Jordanian Commercial banks. A multi-layer feed-forward neural network with backpropagation learning algorithm was used to build up the proposed model. Results: Different representative cases of loan applications were considered based on the guidelines of different banks in Jordan, to validate the neural network model. Conclusion: The results indicated that artificial neural networks are a successful technology that can be used in loan application evaluation in the Jordanian commercial banks.
\end{abstract}

Key words: Business Intelligence (BI), Artificial Intelligence (AI), Artificial Neural Networks (ANN), Backpropagation (BP) algorithm, Credit Scoring (CS)

\section{INTRODUCTION}

Credit loans constitute a cornerstone of the banking industry. The performance of loan contracts in good standing guarantees profitability and stability of a bank. Screening the customer's financial history and financial background is a very significant factor before any credit decision is taken and it is a key process in reducing credit risk. Loan approval will be given to good applicants with low credit risk, whereas high risk applications will be rejected (Limsombunchai et al., 2005). Therefore, banks should control credit management thoroughly. Moreover, the undergoing continuous changes in the business environment, the banking and credit regulations, the marketing strategies of the banks, the competition in banks' lending strategies and the borrowing patterns of customers, demand frequent revision and smart responses. In addition, banking loan decisions require the use of huge and various data and substantial processing time to be able to serve a large number of variables and a variety of different cases related to different customers. Thus, it will be very hard to design or even to use a banking loan model that is capable of tracing all possible and real responses and interactions. Traditional models are unable to exhibit uncertainty in the banking loan environment (Shachmurove, 2002). A set of creditscoring models that correctly classify loan applications have been developed to support traditional judgmental methods (Malhotra and Malhotra, 2003). Furthermore, a challenge for today's commercial banks is their ability to understand large amounts of information and reveal useful knowledge to improve decision-making. Modern bank managers are flooded in data. The sustainability of their banks depends on their capabilities to sift through large volumes of data, to extract useful knowledge and enforce this knowledge in their decisions (Mitchell and Pavur, 2002). An intelligent information system that is based on artificial intelligence will provide managers with added value information, to reduce the uncertainty of the decision outcome and to enhance banking service quality. Thus, the application of new technologies can give a bank a competitive advantage and lead to a higher performance.

Artificial neural networks have been fruitfully used in a variety of business fields including marketing, accounting, management information systems and production management (Cao and Parry, 2009). Most of the studies have used neural networks for predicting future stock behavior, financial crises, bankruptcy, exchange rate and detecting credit card fraud. Furthermore, researchers have used neural networks in

Corresponding Author: Shorouq Fathi Eletter, Department of Management Information Systems,

Faculty of Economics and Administrative Sciences, Al-Zaytoonah University of Jordan,

P.O. Box (130), Amman (11733), Jordan 
modeling market response collective behavior, telecommunication and real estate evaluations. ANNs are also used for analyzing relations among economic and financial phenomena, forecasting, generating time series, optimization and decision making (Shachmurove, 2002). Artificial neural networks have successfully provided effective credit evaluations for supporting granting loans. The Security Pacific Bank was the first bank to announce neural networking system to the public in 1991 (Goonatilake and Treleaven, 1995). Researchers are currently focusing on using neural network classification models and particularly backpropagation neural networks in classifying loan applications into good and bad ones. Customer loans include short and intermediate term loans that are extended through regular business channels to finance the purchase of goods and services for individual consumption (Malhotra and Malhotra, 2003). This research attempts to explore whether using neural networks will provide more accurate personal loan decisions in the Jordanian commercial banks. It will also propose a neural computing model as the basis for a decision support tool in granting or rejecting bank loans. The model will apply banking credit standards to determine the customer who will be eligible for credit approval. A customer will be qualified for credit after meeting the acceptable standards set by the banking institution.

The major risk that a bank faces is the probability of a customer's default. An experienced credit manager should be able to make the right judgment of the probability of default for different types of customers. To have effective managerial control over the credit department requires fast, accurate and real-time information that must be processed in a fair and objective manner. Artificial intelligence technologies have proven to be a fruitful investment in the banking industry, such as neural networks, for their ability to assist in improving the quality of credit decisions to reduce credit risk (Limsombunchai et al., 2005). Thus, artificial neural networks with their capabilities of capturing nonlinear and complex relationships are considered promising techniques in classification problems (Zhang, 2004; Huang et al., 2004).

Traditional judgmental methods and conventional statistical techniques are used in the approval of loan worthiness (Tafti and Nikbakht, 1993). Traditional statistical models such as discriminant analysis and logistic regression usually assume multivariate normality and homoscedastic and these assumptions are often violated in the real world banking data (Giang, 2005; Huang et al., 2004). Moreover, traditional techniques lack the reasoning skills that an experienced loan officer possesses. The current research views the neural network as superior alternatives to the traditional techniques that are used in loan application evaluations.

Problem: Despite the increase in consumer loans defaults and competition in the banking market, most of the Jordanian commercial banks are reluctant to use artificial intelligence software technologies in their decision-making routines. Generally, bank loan officers rely on traditional methods to guide them in evaluating the worthiness of loan applications. A checklist of bank rules, conventional statistical methods and personal judgment are used to evaluate loan applications. Furthermore, a loan officer's credit decision or recommendation for loan worthiness is subjective. After some experience, these officers develop their own experiential knowledge or intuition to judge the worthiness of a loan decision. Given the absence of objectivity, such judgment is biased, ambiguous, nonlinear and humans have limited capabilities to discover useful relationships or patterns from a large volume of historical data (Handzic et al., 2003). Generally, loan applications evaluations are based on a loan officers' subjective assessment. Therefore, a knowledge discovery tool is needed to assist in decision making regarding the application. Furthermore, the complexity of loan decision tools and variation between applications is an opportunity for a neural-computing technology to provide learning capability that does not exist in other technologies. Neural networks are becoming highly granted statistical tools in the banking decision-making process.

Objectives: This study aims to develop a loan decision model using the artificial neural network for the Jordanian commercial banks. This is the first empirical research of its kind in our region that addresses in a systematic way the issue of using artificial neural networks in loan applications evaluations. Neural networks with their capability of capturing nonlinear and complex relationships are a powerful alternative to the conventional forecasting and classification methods. Neural networks are consistent paradigms of the nonparametric approach in financial modeling due to their ability to correctly classify and predict consumer loan defaults.

The purpose of using the neural network algorithm in bank loan decisions is to simplify a loan officer's job, to control it and to achieve more efficiency and productivity (Curry and Moutinho, 1993). This study explores the power of using NNs in banks for loan approval. The general objectives of this study are: 
- To identify neural networks as an enabling tool for evaluating credit applications to support loan decisions

- To outline some of the challenges of using neural networks in the decision-making process for the banking industry in Jordan

Motivation: During the last few decades, banks have relied on the personal assessment of loan risks or on the traditional statistical methods to predict the default of loans instead of using a standardized evaluation tool. The development of neural networks has been seen as one of the most exciting developments in terms of their applicability to business settings. In recent years, neural networks have been moving from research laboratories into the business world and are already at work in the world of banking and finance and else where (Hawley et al., 1990; Huang et al., 2004). Considered by some scholars to be one of the most important technological advances of the last ten years, neural networks are particularly applicable to risk management and forecasting (Huntley, 1991; Huang et al., 2004; Limsombunchai et al., 2005), where the ability to identify complex patterns is crucial to predictions (Zhang, 2004; Huang et al., 2004). The business community's enthusiasm seems to be related to the following:

- Neural networks promise to be a breakthrough in areas where a traditional computer system and statistical methods have difficulty to support the decision making process in today's complex business environment. Neural networks have been used in building intelligent information systems that mimic the way in which humans think. They are suitable for modeling business logical outcomes where variables give unstable signs and interact in a complex non-linear way (Peel and Wilson, 1996)

- Artificial neural networks are promising tools for modeling noisy, inconsistent or incomplete data in different business situations. The application software is self-adjustable for any user and it only requires a representative training set against a standard reference. Then, the ANNs self-adaptive nature will take over (Pollard, 1990)

- Most financial decisions have incomplete information, so neural networks have more applications for such data than do expert systems and advanced statistical methods (Tafti and Nikbakht, 1993)

The advantages of using ANN are as follows:
- Eliminates the necessity of having a linear relationship between input variables and the output. The network simply learns the relationships based on the representative sample data and produces output (the relationship can be linear or nonlinear). This reduces the risk of bias in decision making that might occur if an incorrect functional form or relationship is assumed

- Enables the receipt of output even when presented with input that it has never seen before

- Has the ability to learn from past experience and provide a better output as the system is trained with new examples. This also reduces subjectivity in decision-making. However, the main problem with using the ANN is that it does not tell the system user how it arrived at the results. These obviously depend on the correctness and completeness of the data that has been entered. The system is good at processing and recognizing patterns, but it may be exposed to the risk of producing something out of nothing. Therefore, the data used for training must represent a universal set of parameters

\section{MATERIALS AND METHODS}

This study built a neural network computer program that simulated the physical neural process by which human learning takes place and intuition is formed. The researcher used a multilayer feed-forward neural network model. By using a supervised backpropagation learning algorithm to train the net with a set of cases from the banking sector, the NN will learn by example after being given a sufficient number of input/output cases. The system is programmed without any preexisting rules and structures. It actually learns through experience. A neural network has three layers: the input layer namely, the hidden layer(s) and the output layer. Hidden layers form a processing bridge between source and destination (Shaaf, 1996). The most significant elements in the NN are: The neuron (the processing element) which has an input or more and only one output and the interconnection between the processing elements which are represented by weights (Pollard, 1990).

There are three basic phases of $\mathrm{NN}$ learning, recalling and testing. The system is trained on historical examples of input and output variables. During this learning process, the system learns to recognize patterns by constructing the relationship between inputs and outputs coming up with the final output. Then, a comparison between the actual and desired output occurs and errors are calculated (Shaaf, 1996). The errors are used to adjust the weights so that the 
variation between source values and target values is minimized. The concept of learning by example is the most important concept for training the network. Since the neural network provides output on the basis of its prior training, the training must be representative of the targeted population. Then the memorized pattern can be used in the testing phase to examine the accuracy of the network. This procedure is based on the neural network methodology for designing a credit-scoring model that can be used by Jordanian commercial banks for decision making regarding loans.

Banking scoring system: Credit scoring is the key in reducing the credit risk on loan applications. This includes determining the financial strength of the borrowers, approximating the probability of default and reducing the risk of non-payment to an acceptable level. Credit scoring employs a model of evaluating a set of variables to determine creditworthiness of similar loan applications. A combined credit score is calculated relative o the value of each variable. The result will be compared to a cutoff point. If the score exceeds the cutoff point, the application is approved; if not, the application is rejected (Tafti and Nikbakht, 1993). However, the credit scoring cannot totally remove the human factor. The selection of cutoff scores is a subjective decision. The evaluation of applications that have scores between the accepted scores and rejected scores is an individual judgment (Malhotra and Malhotra, 2003). Credit scoring models have the potential in reducing the inconsistency of credit decisions and adding effectiveness to the lending risk assessment practice. Moreover, in addition to their role in the loan approval process, credit scoring models assist on loan pricing, loan monitoring, determining the amount of credit, credit risk management and the assessment of loan portfolio risks. Credit scoring is widely applied in consumer lending mainly in credit cards and mortgage lending (Limsombunchai et al.,
2005). However, there are some problems associated with scoring consumer credits:

- The inability to discriminate due to the complexity of the data

- $\quad$ Some scoring systems do not work in practice and become overtaken by change (Walker et al., 1995)

Credit scoring has not been commonly used in the Jordanian commercial banks. Generally, the bank employs loan officers to make credit decisions or recommendations for the bank. The lending officer usually makes approval for applications that are worthy of giving a loan. These officers are given some guidelines to direct them in evaluating the worthiness of loan applications depending on the borrowers' characteristics. This practice is inefficient, inconsistent and non-uniform. Therefore, the main purpose of this research is to develop a lending decision model for the product of personal loans in Jordanian commercial banks using a back-propagation multi-layer feedforward neural network.

A proposed neural network model for loan decisions: Designing a neural network successfully relies on a clear understanding of the problem and on deciding upon most influential input variables. The banking data set had 140 personal loan applications. Of these, 94 cases were used in the training and 46 were used in the testing. Both training and testing data sets contained half-good applications and half-bad applications. There are 11 influential variables over the loan decision. The definition and recoding of the variables are given in Table 1. On the other hand; the output for the neural network was 1 for good applications or 0 for bad applications. The procedure of designing a neural network model is a logical process. The process was not a single-pass one, but it required going back to previous steps several times.

Table 1: Loan decision factors

\begin{tabular}{lll}
\hline Variable code & Variable description & Variable recoding \\
\hline $\mathrm{X}_{1}$ & Age & 1 if the applicants age is accepted, 0 otherwise. \\
$\mathrm{X}_{2}$ & Account type & 1 for payroll account, 2 for self account \\
$\mathrm{X}_{3}$ & Income & 0 if income $<400,1$ for $(400 \leq$ income $<1000)$ and 2 for (income $\geq 1000)$. \\
$\mathrm{X}_{4}$ & Nationality & Nationality 1 for Jordanian resident and 0 otherwise. \\
$\mathrm{X}_{5}$ & Residency & 1 for resident and 0 otherwise \\
$\mathrm{X}_{6}$ & Company's type & 1 if the company is accredit by the bank and 0 otherwise \\
$\mathrm{X}_{7}$ & Guarantor & 1 if the applicant has guarantor and 0 otherwise. \\
$\mathrm{X}_{8}$ & Job experience & 0 if (exp. $<6$ months), $1(6$ months $\leq$ exp. $<2$ years) and 2 for (exp. $\geq 2$ years) \\
$\mathrm{X}_{9}$ & Debt balance ratio & 1 for good DBR and 0 otherwise \\
$\mathrm{X}_{10}$ & Social security & 1 if the applicant has social security account and 0 otherwise. \\
$\mathrm{X}_{11}$ & Loan size & Between 1000 and 35000 for payroll account type and between 1000 and 25000 for self-account.
\end{tabular}




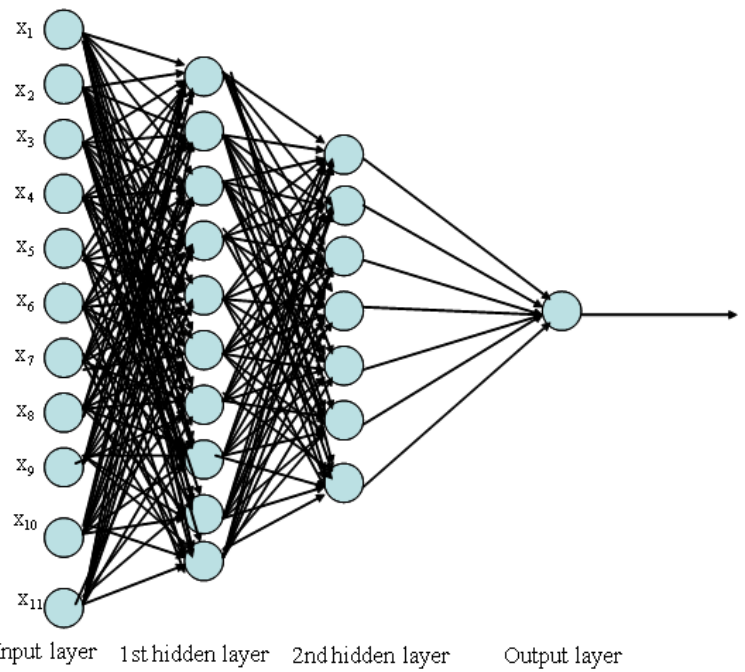

Fig. 1: Topology of the neuro-loan model. Source: Prepared by researcher

Defining the network parameters: Deciding on the optimal parameters for the MLFN model is a critical issue. The best model is one that has the combination of parameters that minimizes the mean squared error. At the beginning, the researchers selected an initial configuration (one hidden layer with a number of hidden neurons and one neuron in the hidden layer). After conducting a number of experiments with each model, the researcher retained the best network. On each experiment if the network does not achieve an acceptable performance level, we tried adding more neurons to the hidden layer. If this did not help, we tried adding an extra hidden layer. In brief, a series of repeated trials were applied in order to reach the optimal set of parameters. The network was given a fixed number of training epochs 10000; the learning rate was set to 0.2 , the momentum is 0.9 , the $\log$ sigmoid is the transfer function and the gradient descent is the learning algorithm.

Number of hidden neurons: The objective of the first successive trials was to hit upon the optimal number of hidden neurons that would enable the network to evaluate loan applications with the best performance. The process started by using one hidden layer with six hidden neurons that is equal to the average of the input neurons and the output neurons, then the number of hidden neurons was increased incrementally until reaching 20 neurons and decreased to 1 . Most cases needed over 10000 epochs to converge. Furthermore, the new set of trails increased the number of hidden layers to two and three hidden layers with a different number of neuron combinations in order to decide on the best set of hidden layers and neurons. A multi-layer feed-forward neural network as shown in (Fig. 1) with two hidden layers with ten neurons in the first hidden and seven neurons in the second hidden had the best speed time at the fixed threshold level of 0.05 .

Learning rate: This research carried out different trials to decide on a learning rate that successfully directed the degree of weight modification during the training epochs. For each trial, there were different runs of the network. The error threshold was set at 0.05 levels and the number of epochs was set at 10000 . Then, the average epochs needed to reach 0.05 were calculated. The training would stop whenever the performance of the network reached below 0.05 or when the training epochs reached 10000 even if the goal was not met. Then it was determined to set the learning rate to 0.2 while trying to look for the optimal hidden architecture. After things started to be clearer, the learning rate was set to 0.1 and 0.05 to compare the results at the different learning rate levels. Surprisingly, the two hidden layers neural network with ten and seven neurons did not converge at that learning level.

Momentum: Momentum allows a network to respond to new trends in the error surface. It also allows the network to disregard small features in the error surface. A number of trials were made to find the momentum that helps the network local minima. For each trial, there were different runs while the error threshold was set at level 0.05 . The network stops training when the error percentage reaches a level below the threshold. Setting the momentum to 0.9 has contributed to the network's speed of learning. The momentum was set on a level lower than 0.9 , the convergence time increased. When the momentum was set at one, the goal was not met at 10000 epochs.

\section{RESULTS}

Network training: First the networks' weights are initialized, consequently the network is prepared for training. The training cases are used to adjust the weights through minimizing the prediction made by the network. The back-propagation gradient descentlearning algorithm uses the performance function in order to trace the best set of weights that minimizes the average mean squared error. The algorithm allocates the error backward through the network's layers successively. Furthermore, the algorithm develops iteratively, through a number of epochs and uses the error to adjust the weights in the direction in which the performance function decreases quickly. The training stopped when the error reached 0.0499948 performance level and about 4670 epochs shown in Fig. 2. 
Am. J. of Economics and Business Administration 2 (1): 27-34, 2010

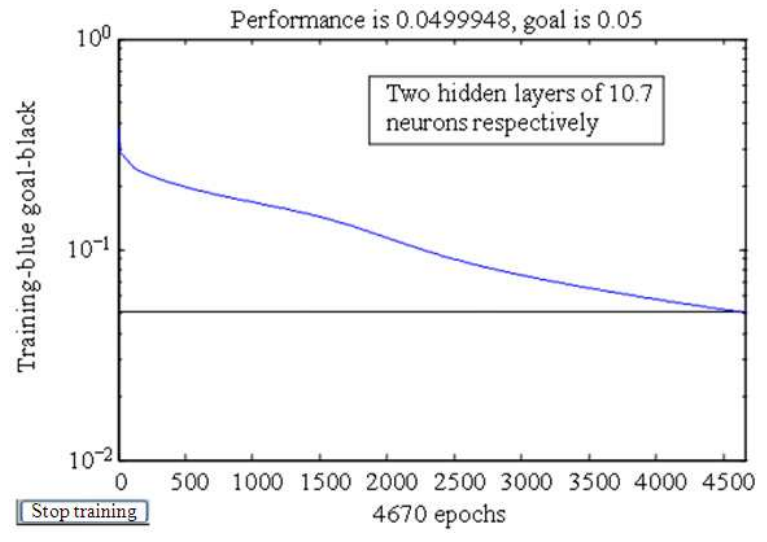

Fig. 2: The process of training the neural network

Network testing: Testing the network is necessary in order to examine its ability to classify the testing data correctly. Testing starts after the training has been completed. The network was simulated on the testing set (i.e., cases the network has not seen before). The results were very good; the network was able to classify $95 \%$ of the cases in the testing set.

\section{DISCUSSION}

Currently, the efficient management of credit loans is very significant for the banking industry in Jordan in particular and the banking industry in general. The massive increase in computer power and the reasonable cost of purchasing neural network software are employed to resolve complex business problems. This study proposed a decision support system that is able to adapt to the dynamic changes in the financial business environment. The proposed system will assist Jordanian banks in personal credit evaluation in order to reduce the risk of customers who default. The commercial banks in Jordan rely on loan officers to make credit decisions. There might be some cases where the customers either through personal contacts or through friends know loan officers, which might affect their evaluation capabilities. Using neural networks in loan evaluation will reduce any bias or emotional intention that can distort the decision process. Hence, the cost of loan processing will be reduced; also it will reduce personal judgment which will enhance the quality of customer service. On the other hand, there are cases were customers need a loan badly, but they might not be worthy of a loan because their application does not meet the required criteria. Their interaction with loan officers will enhance their other qualifications and they will be able to get the loan. That means the human touch in the service industry has a special flavor that might enhance its growth in some cases. Such a program will not eliminate the human interaction but it is proposed to enhance the decision process. Even though there are some cases needing human intervention, generally, the neural networks outperformed conventional methods concerning a loan's risk prediction. Moreover, the research findings correspond with research by Handzic et al. (2003); Malhotra and Malhotra (2003) and Mitchell and Pavur (2002) indicating that neural networks are a successful technology that can be used in loan application evaluations. The results also showed that the use of more training cases means better results.

\section{CONCLUSION}

Although the credit decision approval in the Jordanian banks has been subjective and it is up to the loan officer in most cases, banks can improve their credit analysis methods through utilizing artificial intelligence technologies such as neural networks. This study has proposed a new technique to evaluate loan application as a decision support system in the field of banking credit system; the proposed neural network model was designed to represent the most influential factors to the credit decision in Jordanian Banks. These factors were taken from the guidelines that loan officers use in the Jordanian banks. Besides, banks can customize the system according to their specific strategies. Furthermore, the high accuracy of this model proves that neural networks can be useful for classifying loan applications.

Recommendations: The research objective was to design a decision support tool to classify loan applications into good or bad. The experimental results showed that MLFF neural network is an effective tool in classifying loan applications at a high accuracy level. Therefore, the implementation of such technology in Jordanian banks can be helpful for the decision making process. However, to gain more trust in these technologies, banks can use more cases of successful loan applications and bad applications from their databases to enhance the accuracy of the proposed model. The network with its adaptive nature can accommodate new data without reprocessing old ones. Successful unbiased credit analysis is very significant to determine the profitability and stability of the bank and it reduces its credit risk.

Study limitations: In general, identifying the major variables that influence the loan decision approval was 
a critical issue. There are 13 commercial banks with different strategies. It was very difficult to obtain a comprehensive set of actual data from local commercial banks. A bank considers such information confidential and it should be hidden from competitors (other banks). Another limitation in applying a neural network model was the determination of the optimal set of training parameters including the network topology. Also, the neural network needed long training times. Too many epochs were required to train the network. Building the neural network model was a process of trial and error.

Suggestions for future studies: This research introduced a proposed model using neural networks technologies to help loan officers make better decisions when evaluating personal loan applications. As a guideline for further research, this model could be extended to include other types of loans. At the same time, it can include a comparative study of other types of neural networks with a different learning algorithm. To enhance the acceptance of these technologies, researchers should provide users with figures to come over the black box nature of neural networks. A further field of research is the application of genetic algorithm to the network design. Genetic algorithm may improve the process of finding better input data set parameters. Genetic algorithms take on an evolutionary approach to modify the neural network weights. A random generated set of initial weights called population evolves through successive operations includes reproduction, crossover and mutation. Each synthesized network will be evaluated using the back propagation. Moreover, genetic algorithms have a built-in-bias towards keeping good arrangements in the next generation. The search for good configuration proceeds in parallel ways. Therefore, increases the possibility of successful convergence (Cao and Parry, 2009; Malhotra and Malhotra, 2003). Furthermore, providing simple graphical user interface to the network model will provide the end user with greater trust of the net performance. Moreover, researchers can modify the proposed model to be available to customers as an extension to ATM services. Besides, the emergence of E-banking has made everything at customers' fingertips. The E-banking system can easily utilize neuro-models within an effective interface. Customers can make use of this innovation as an online credit evaluation. Furthermore, researchers can merge the system with expert systems in order to give feedback to customers in the case of credit disapproval.

\section{REFERENCES}

Cao, Q. and M. Parry, 2009. Neural network earning per share forecasting models: A comparison of backward propagation and genetic algorithm. Dec. Support Syst., 47: 32-41. DOI: 10.1016/j.dss.2008.12.011

Curry, B. and L. Moutinho, 1993. Using advanced computing techniques in banking. Int. J. Bank Market., $\quad$ 11: 39-46. $\quad$ DOI: $10.1108 / 02652329310045756$

Giang, H., 2005. Using neural network in predicting corporate failure. J. Soc. Sci., 1: 199-202.

Goonatilake, S. and P. Treleaven, 1995. Intelligent Systems for Finance and Business. 1st Edn., John Wiley and sons, USA, ISBN : 0471 944041, pp: 62.

Handzic, M., F. Tjandrawibawa, and J. Yeo, 2003. How/neural networks can help loan officers to make better informed applications decisions. http://informingscience.org/proceedings/IS2003Pro ceedings/docs/024Handz.pdf

Hawley, D.D., J.D. Johnson and D. Raina, 1990. Artificial neural systems: A new tool for financial decision-making. Fin. Anal. J., 46: 63-72. DOI: 10.2307/2F4479380

Huang, W., K.K. Lai, Y. Nakamori and S. Wang, 2004. Forecasting foreign exchange rates with artificial neural networks: A review. Int. J. of Inform. Technol. Dec. Mak., 3: 145-165. DOI: 10.1.1.121.8174

Huntley, D.G., 1991. Neural nets: An approach to the forecasting of time series. Soc. Sci. Comput. Rev., 9: 27-38. DOI: 10.1177/089443939100900104.

Limsombunchai, V., C. Gan and M. Lee, 2005. An analysis of credit scoring for agricultural loans in Thailand. Am. J. Applied Sci., 2:1198-1205.

Malhotra, R. and D.K. Malhotra, 2003. Evaluating consumer loans using neural networks. Omega, 31: 83-96. DOI: 10.1016/S0305-0483(03)00016-1

Mitchell, D. and R. Pavur, 2002. Using modular neural networks for business decisions. Manage. Dec., 40: 58-63. DOI: 10.1108/00251740210413361.

Peel, M. and N. Wilson, 1996. Neural network simulation: A new approach to risk assessment and business forecasting. Manage. Res. News, 19: 50-54. DOI: 10.1108/eb028477

Pollard, A., 1990. What are Neural Networks? Sensor Rev., 10: 115-116. DOI: 10.1108/eb007822

Shaaf, M., 1996. A neural network and econometric comparison of the relative importance of fiscal and monetary actions. Stud. Econ. Fin., 17: 69-87. DOI: $10.1108 / \mathrm{eb} 028727$ 
Shachmurove, Y., 2002. Applying artificial neural networks to business, economics and finance. http://ideas.repec.org/p/cla/penntw/5ecbb5c20d3d5 47f357aa130654099f3.html

Tafti, M.H.A. and E. Nikbakht, 1993. Neural networks and expert systems: new horizons in business finance applications. Inform. Manage. Comput. Secur., 1 : 22-28. DOI: $10.1108 / 096852293 / 0026502$.
Walker, R. F., E.W. Haasdijk and M.C. Genets, 1995. Credit Evaluation Using a Genetic Algorithm. In: Intelligent System for Finance and Business, Goonatilake, S. and P. Treleaven (Eds.). John Wiley and Sons, ISBN: 0471 944041, pp: 39-59.

Zhang, G.P., 2004. Neural Networks in Business Forecasting. 1st Edn., Idea Group Inc., ISBN: 159140-176-3, pp: 1-41. 